\title{
LIFTINGS OF FUNCTIONS WITH VALUES IN A COMPLETELY REGULAR SPACE
}

\author{
G. A. EDGAR ${ }^{1}$ AND MICHEL TALAGRAND
}

\begin{abstract}
Let $T$ be a completely regular space, let $(\Omega, \mathcal{F}, \mu)$ be complete probability space, and let $\rho: \mathfrak{L}^{\infty}(\mu) \rightarrow \mathfrak{L}^{\infty}(\mu)$ be a lifting. If $f: \Omega \rightarrow T$ is a Baire measurable function, must there exist a function $\tilde{f}$ with almost all of its values in $T$, such that $\rho(h \circ f)=h \circ \tilde{f}$ for all bounded continuous functions $h$ on $T$ ? If $T$ is strongly measure-compact, then the answer is "yes". If $T$ is not measure-compact, then the answer is "no". This shows that a lifting is not always the best method for the construction of weak densities for vector measures.
\end{abstract}

Let $T$ be a completely regular Hausdorff space, and let $C_{b}(T)$ be the set of bounded continuous real-valued functions on $T$. Thus $T$ is homeomorphically embedded in $\mathbf{R}^{C_{b}(T)}$ (in the product topology) by the map $\Phi: T \rightarrow \mathbf{R}^{C_{b}(T)}$ defined by $\Phi(t)(h)=h(t), t \in T, h \in C_{b}(T)$. We will identify $T$ with its image $\Phi[T]$. Any bounded continuous function on $T$ extends to a bounded continuous function on $\mathbf{R}^{C_{b}(T)}$, so the Baire sets in $T$ are the intersections of $T$ with the Baire subsets of $\mathbf{R}^{C_{b}(T)}$.

Let $(\Omega, \mathscr{F}, \mu)$ be a complete probability space. A function $f: \Omega \rightarrow T$ is Baire measurable if and only if $h \circ f$ is measurable for all $h \in C_{b}(T)$. Let $\rho$ be a lifting for $\mathcal{L}^{\infty}(\Omega, \mathscr{F}, \mu)$ (see [3, p. 34] for the definition). Define $\rho^{\prime}(f): \Omega \rightarrow \mathbf{R}^{C_{b}(T)}$ by $\rho^{\prime}(f)(\omega)(h)=\rho(h \circ f)(\omega), \omega \in \Omega, h \in C_{b}(T)$. Then $\rho^{\prime}(f)$ is Baire measurable (in fact Borel measurable [3, p. 52]), and for any $h \in C_{b}\left(\mathbf{R}^{C_{b}(T)}\right)$, we have $h \circ \rho^{\prime}(f)=$ $h \circ f$ a.e. and $\rho(h \circ f)=h \circ \rho^{\prime}(f)$ everywhere [3, §IV. 5].

Under what circumstances does the lifting $\rho^{\prime}(f)$ of $f$ have its values in $T \subseteq$ $\mathbf{R}^{C_{b}(T)}$ ? A well-known sufficient condition is that $f[\Omega]$ be relatively compact in $T$ [3, p. 52]. In fact, for nonatomic $\mu$, if $\rho$ varies over all liftings for $\mathfrak{L}^{\infty}(\mu)$, then $\rho^{\prime}(f)(\omega)$ varies over all values in the essential range $\left\{y \in \mathbf{R}^{C_{b}(T)}: \mu\left(f^{-1}(U)\right)>0\right.$ for all neighborhoods $U$ of $y$, which is a compact subset of $\mathbf{R}^{C_{b}(T)}$. Thus, in order that $\rho^{\prime}(f)[\Omega] \subseteq T$ for all liftings $\rho$, it is necessary and sufficient that the essential range of $f$ be compact in $T$. This can be seen as follows. Let $y_{0}$ belong to the essential range of $f$, and choose $\omega_{0} \in \Omega$ with $\mu\left(\left\{\omega_{0}\right\}\right)=0$. Let $\mathcal{Q}$ be a maximal filter of sets from $\mathcal{F}$ of positive measure that includes $\left\{f^{-1}(U): U\right.$ is an open neighborhood of $\left.y_{0}\right\}$. Then any lifting $\rho$ for $\mathcal{L}^{\infty}(\mu)$ remains a lifting when redefined at $\omega_{0}$ as the limit along $\mathscr{U}$ :

$$
\rho(g)\left(\omega_{0}\right)=\lim _{\omega, \mathcal{Q}} g(\omega), \quad g \in \mathcal{L}^{\infty}(\mu) .
$$

Then $\rho^{\prime}(f)\left(\omega_{0}\right)=y_{0}$.

Received by the editors August 23, 1978 and, in revised form, April 26, 1979.

AMS (MOS) subject classifications (1970). Primary 46G15.

${ }^{1}$ Supported in part by National Science Foundation grant number MCS77-04049.

(c) 1980 American Mathematical Society $0002-9939 / 80 / 0000-0112 / \$ 02.25$ 
In this paper we are interested in a related question: Under what circumstances does $\rho^{\prime}(f)$ have almost all of its values in $T$ ?

We will be considering three classes of measures on a completely regular space $T$. First $P_{\sigma}(T)$ denotes the set of probability measures on the $\sigma$-algebra of Baire sets in $T$. Second, $P_{\tau}(T)$ denotes the set of $\tau$-smooth probability measures, i.e. measures $\lambda \in P_{o}(T)$ such that $\int h_{\alpha} d \lambda \rightarrow 0$ for any net $h_{\alpha} \in C_{b}(T)$ which decreases pointwise to 0. Finally, $P_{t}(T)$ denotes the set of tight probability measures, i.e. measures $\lambda \in P_{\sigma}(T)$ such that, for every $\varepsilon>0$, there is a compact $K \subseteq T$ with $\inf \{\lambda(B)$ : $B \supseteq K, B$ is a Baire set $\} \geqslant 1-\varepsilon$. (These are the measures which extend to Radon measures on $T$.)

A completely regular space $T$ is called universally Radon measurable iff $P_{\tau}(T)=$ $P_{t}(T)$ (justification for this terminology can be found in [8] or in [4]). A space $T$ is called measure-compact iff $P_{\sigma}(T)=P_{\tau}(T)$ [5]. A space $T$ is called strongly measure-compact iff $P_{\sigma}(T)=P_{t}(T)$ [5], i.e. $T$ is both universally Radon measurable and measure-compact. Examples of spaces $T$ which do and do not have these properties can be found in [5].

THEOREM 1. Let $T$ be a completely regular Hausdorff space. Then $\left(a^{\prime}\right) \Rightarrow(b) \Rightarrow$ $\left(a^{\prime \prime}\right)$.

(a') $T$ is strongly measure-compact.

(b) If $(\Omega, \mathcal{F}, \mu)$ is a complete probability space, if $\rho$ is a lifting for $\mathfrak{L}^{\infty}(\mu)$, and if $f$ : $\Omega \rightarrow T$ is Baire measurable, then $\rho^{\prime}(f)(\omega) \in T$ for almost all $\omega \in \Omega$.

(a") $T$ is measure-compact.

If $T$ is universally Radon measurable, then the three conditions are equivalent.

With the same proof (but twice as many definitions) we can obtain a more general result.

Let $T$ be a completely regular space, and let $H \subseteq C_{b}(T)$ be a set of functions which determines the topology in $T$, i.e. $\Re_{H}=\left\{h^{-1}(U): h \in H, U \subseteq \mathbf{R}\right.$ is open $\}$ is a subbase for the topology. We will assume $H$ is a uniformly closed algebra which contains the constants. (For example, if $T \subseteq S$ where $S$ is completely regular, let $H$ be the set of functions in $C_{b}(T)$ which have continuous extensions to $S$.) Then $\Phi: T \rightarrow \mathbf{R}^{H}$ defined by $\Phi(t)(h)=h(t)$ identifies $T$ homeomorphically with a subset of $\mathbf{R}^{H}$. Let $\mathscr{B}_{H}$ be the $\sigma$-algebra generated by $\Re_{H}$, so that $\mathscr{B}_{H}$ consists of the sets $T \cap B$, where $B$ is a Baire set in $\mathbf{R}^{H}$. In general, not every bounded continuous function on $T$ can be extended to $\mathbf{R}^{H}$, and $\mathscr{B}_{H}$ may be strictly smaller than the $\sigma$-algebra of Baire sets of $T$.

Let $(\Omega, \mathscr{F}, \mu)$ be a complete probability space, and let $\rho$ be a lifting for $\mathcal{L}^{\infty}(\mu)$. If $f: \Omega \rightarrow T$ is $\mathscr{B}_{H}$-measurable, then $\rho^{\prime}(f)(\omega)(h)=\rho(h \circ f)(\omega), \omega \in \Omega, h \in H$, defines $\rho^{\prime}(f): \Omega \rightarrow \mathbf{R}^{H}$.

Let $\lambda$ be a probability measure on $\Re_{H}$. Then $\lambda$ is called tight iff, for every $\varepsilon>0$, there is a compact set $K \subseteq T$ such that $\inf \left\{\lambda(B): B \in \mathscr{B}_{H}, B \supseteq K\right\}>1-\varepsilon$; or, equivalently, iff $\lambda$ extends to a Radon measure on $T$. Also, $\lambda$ is called $\tau$-smooth iff for any net $f_{\alpha} \in H$ which decreases to 0 , we have $\lim \int f_{\alpha} d \lambda=0$. (Since $H$ generates the topology of $T$, a measure $\lambda$ on $\mathscr{B}_{H}$ is $\tau$-smooth if and only if it extends to a $\tau$-smooth measure on the Baire sets of $T$.) 
TheOREM 2. Let $T, H, \mathfrak{B}_{H}$ be as above. Then $\left(\mathrm{a}^{\prime}\right) \Rightarrow(\mathrm{b}) \Rightarrow\left(\mathrm{a}^{\prime \prime}\right)$.

(a') Every probability measure on $\mathscr{B}_{H}$ is tight.

(b) If $(\Omega, \mathcal{F}, \mu)$ is a complete probability space, if $\rho$ is a lifting for $\mathfrak{L}^{\infty}(\mu)$, and if $f$ : $\Omega \rightarrow T$ is $\mathscr{B}_{H^{-}}$-measurable, then $\rho^{\prime}(f)(\omega) \in T$ for almost all $\omega \in \Omega$.

( $\left.a^{\prime \prime}\right)$ Every probability measure on $\mathscr{B}_{H}$ is $\tau$-smooth.

If $T$ is universally Radon measurable, then the three conditions are equivalent.

Proof. $\left(\mathrm{a}^{\prime}\right) \Rightarrow(\mathrm{b})$. Let $(\Omega, \mathscr{F}, \mu), \rho$, and $f$ be given. Let $\lambda=f(\mu)$ be the image measure, defined by $\lambda(B)=\mu\left(f^{-1}(B)\right)$ for $B \in \mathscr{B}_{H}$, or by $\int h d \lambda=\int h \circ f d \mu$ for $h \in H$. By the assumption ( $\left.\mathrm{a}^{\prime}\right), \lambda$ is tight. Let $\varepsilon>0$ be given. There is a compact set $K \subseteq T$ so that $\lambda(B) \geqslant 1-\varepsilon$ for every $B \in \mathscr{B}_{H}$ with $B \supseteq K$. Then

$$
C=\cap\left\{\rho(A): A=f^{-1}(B), B \in \mathscr{B}_{H}, B \supseteq K\right\}
$$

is in $\mathscr{F}, \mu(C) \geqslant 1-\varepsilon$, and $\rho(C) \subseteq C\left[3\right.$, p. 40]. We claim that $\rho^{\prime}(f)(\omega) \in K$ for all $\omega \in \rho(C)$. Indeed, suppose $y \in \mathbf{R}^{H} \backslash K$. Then there is a continuous function $g$ : $\mathbf{R}^{H} \rightarrow[0,1]$ with $g(y)=1$ and $g(t)=0$ for all $t \in K$. Now ([3, p. 52]; $f[\Omega]$ is relatively compact in $\mathbf{R}^{H}$ ) since $g \circ f=0$ a.e. on $C, g \circ \rho^{\prime}(f)=0$ everywhere on $\rho(C)$. So, if $\omega \in \rho(C)$, we have $\rho^{\prime}(f)(\omega) \neq y$. This shows $\rho^{\prime}(f)[\rho(C)] \subseteq K \subseteq T$. Now $\mu(\rho(C)) \geqslant 1-\varepsilon$, and $\varepsilon$ was chosen arbitrarily, so $\rho^{\prime}(f)(\omega) \in T$ for almost all $\omega \in \Omega$.

(b) $\Rightarrow\left(\mathrm{a}^{\prime \prime}\right)$. Let $\lambda$ be a probability measure on $\mathscr{B}_{H}$. Let $\Omega=T$, let $(\Omega, \mathscr{F}, \mu)$ be the completion of $\left(T, \mathscr{B}_{H}, \lambda\right)$ and let $f: \Omega \rightarrow T$ be the identity function. Suppose $\rho$ is a lifting for $\mathcal{L}^{\infty}(\mu)$. By hypothesis $(\mathrm{b}), \rho^{\prime}(f)(\omega) \in T$ for almost all $\omega \in \Omega$. Let $h_{\alpha} \in H$ decrease pointwise to 0 on $T$. Let $h_{\alpha}^{\prime} \in C_{b}\left(\mathbf{R}^{H}\right)$ be an extension of $h_{\alpha}$. Then $h_{\alpha}^{\prime} \circ \rho^{\prime}(f)$ decreases to 0 a.e., and $\rho\left(h_{\alpha} \circ f\right)=h_{\alpha}^{\prime} \circ \rho^{\prime}(f)$, so $h_{\alpha}^{\prime} \circ \rho^{\prime}(f)$ decreases everywhere and

$$
\lim \int h_{\alpha}^{\prime} \circ \rho^{\prime}(f) d \mu=\int \lim h_{\alpha}^{\prime} \circ \rho^{\prime}(f) d \mu=0
$$

$[3$, p. 40]. Thus

$$
\begin{aligned}
\lim \int h_{\alpha} d \lambda & =\lim \int h_{\alpha} \circ f d \lambda \\
& =\lim \int h_{\alpha} \circ f d \mu=\lim \int \rho\left(h_{\alpha} \circ f\right) d \mu=0
\end{aligned}
$$

Therefore $\lambda$ is $\tau$-smooth.

Finally, if $T$ is universally Radon measurable, we have $\left(a^{\prime \prime}\right) \Rightarrow\left(a^{\prime}\right)$. If $\lambda$ is a probability measure on $\mathscr{B}_{H}$, then by assumption $\left(\mathrm{a}^{\prime \prime}\right), \lambda$ is $\tau$-smooth. But then $\lambda$ extends to a $\tau$-smooth Baire measure on $T$, which is tight since $T$ is universally Radon measurable. Hence $\lambda$ is tight.

Neither of the implications in Theorem 1 can be reversed in general, as the following two examples show.

EXAMPLE 3. $\left(a^{\prime \prime}\right) \neq(b)$. Write $\tau$ for the usual topology on $[0,1]$, and $\lambda$ for Lebesgue measure on $[0,1]$. By the well-ordering theorem [7, Theorem 5.3], there is a set $M \subseteq[0,1]$ such that $F \cap M \neq \varnothing$ and $F \backslash M \neq \varnothing$ for all uncountable closed 
sets $F$ in $[0,1]$. In particular, $M$ has inner measure 0 and outer measure 1 . Let $\Omega=M$, and let $\mu$ be the measure induced on $\Omega$ by $\lambda$, i.e. $\mu(\Omega \cap A)=\lambda(A)$ for any Lebesgue measurable set $A$.

The topological space $T$ will be $[0,1]$ together with the toplogy $\tau^{\prime}$ whose open sets are all sets of the form $G \cup P$, where $G$ is $\tau$-open and $P$ is any subset of $M$. Equivalently, $\tau^{\prime}$ is the topology determined by the set $\left\{\chi_{\{s\}}: s \in M\right\} \cup\{i\}$ of real-valued functions on $T$, where $i$ is the identity function. A set $A \subseteq T$ is $\tau^{\prime}$-closed if and only if it can be written in the form $A=F \backslash P$, where $F$ is $\tau$-closed and $P \subseteq M$. In particular, if $A$ is $\tau^{\prime}$-closed and $A \subseteq M$, then $A \subseteq F \subseteq M$ for some $\tau$-closed set $F$ which (being disjoint from [0, 1] $\backslash M$ ) must be countable.

Now consider a set $A$ which is a $\tau^{\prime}$-(open $F_{\sigma}$ )-set. Since $A$ is $\tau^{\prime}$-open, $A=G \cup P$, where $G$ is $\tau$-open and $P \subseteq M \backslash G$. Also, $A=\cup{ }_{n=1}^{\infty} A_{n}$, where $A_{n}$ is $\tau^{\prime}$-closed, so $A_{n} \cap P=A_{n} \backslash G$ is $\tau^{\prime}$-closed and contained in $M$, hence countable. Then $P=$ $\cup_{n=1}^{\infty}\left(P \cap A_{n}\right)$ is countable.

Define $f: \Omega \rightarrow T$ by $f(\omega)=\omega$. If $A$ is a $\tau^{\prime}$-(open $F_{\sigma}$ )-set, then $A=G \cup P$, where $G$ is $\tau$-open and $P$ is countable, so $A$ is Lebesgue measurable, and hence $f^{-1}(A)=$ $\Omega \cap A$ is $\mu$-measurable. This shows that $f$ is a Baire measurable function from $\Omega$ to $T$. Suppose (for purposes of contradiction) that $\rho^{\prime}(f)(\omega) \in T$ for almost all $\omega \in \Omega$. Now for $\omega \in \Omega$, the function $\chi_{\{\omega\}}$ is a continuous function on $T$, and $\chi_{\{\omega\}} \circ f=0$ a.e., so $\chi_{\{\omega\}} \circ \rho^{\prime}(f)=0$ everywhere, so $\rho^{\prime}(f)(\omega) \neq \omega$. On the other hand, the identity function $i: T \rightarrow \mathbf{R}$ is continuous on $T$, so $i \circ f=i \circ \rho^{\prime}(f)$ a.e., that is, $\rho^{\prime}(f)(\omega)=\omega$ for almost all $\omega \in \Omega$. This contradiction shows that $\rho^{\prime}(f)(\omega)$ is not in $T$ for almost all $\omega$.

Finally, we claim that $T$ is measure-compact. It suffices to show that $T$ is Lindelof [9, p. 175]. Let $\left\{A_{i}: i \in I\right\}$ be a cover of $[0,1]$ by $\tau^{\prime}$-open sets. Write $A_{i}=G_{i} \cup P_{i}$, where $G_{i}$ is $\tau$-open and $P_{i} \subseteq M$. Let $G=\cup_{i \in I} G_{i}$. There is a countable set $I_{1} \subseteq I$ with $G=\cup_{i \in I_{1}} G_{i}$, since $(G, \tau)$ is separable and metrizable. Now $[0,1] \backslash G=\left(\cup_{i \in I} A_{i}\right) \backslash G \subseteq \cup_{i \in I} P_{i} \subseteq M$, and $[0,1] \backslash G$ is $\tau$-closed, so it is countable. Thus, there is a countable set $I_{2} \subseteq I$ such that $[0,1] \backslash G \subseteq \cup_{i \in I_{2}} P_{i}$. Thus $\left\{A_{i}: i \in I_{1} \cup I_{2}\right\}$ is a countable subcover of $[0,1]$. This shows that $\left(T, \tau^{\prime}\right)$ is Lindelof.

Example 4. (b) $\neq$ (a). Let $M, \mu$ be as in Example 3. Let $T$ be $M$ with the usual topology $(\tau)$. Then $\mu$ is a Baire measure on $T$ which is not tight. This shows that $T$ is not strongly measure-compact. But if $(\Omega, \mathscr{F}, \lambda)$ is any complete probability space, $\rho$ is a lifting for $\mathcal{L}^{\infty}(\lambda)$, and $f: \Omega \rightarrow T$ is Baire measurable, then (since the inclusion map $T \rightarrow \mathbf{R}$ is continuous and bounded) $\rho^{\prime}(f)=f$ a.e., so $\rho^{\prime}(f)(\omega) \in T$ for almost all $\omega \in \Omega$.

The work in this paper started from the following question of A. Goldman: Is lifting the best way to construct weak densities for vector measures? (See [2].) The methods. used in Theorem 2 will also prove the following.

THEOREM 5. Let $(\Omega, \mathcal{F}, \mu)$ be a complete probability space, let $E$ be a locally convex space, let $\mathrm{m}: \mathcal{F} \rightarrow E$ be a vector measure with bounded $\mu$-average range, and let $\lambda$ be the associated cylindrical measure. Then $(a) \Rightarrow(b) \Rightarrow(c) \Rightarrow(d)$.

(a) $\lambda$ is (the restriction of) a Radon measure. 
(b) Every lifting gives a density for $\mathbf{m}$.

(c) There is a lifting which gives a density for $\mathbf{m}$.

(d) $\lambda$ is $\tau$-smooth.

Our last example illustrates a situation where weak densities exist, but cannot be obtained from liftings.

EXAMPLE 6. Suppose $E$ is a Banach space with the following three properties. (a) Every vector measure with bounded average range in $E$ has a weak density. (b) Every bounded scalarly measurable function with values in $E$ is Pettis integrable. (c) There is a bounded scalarly measurable function $f$ with values in $E$ which is not weakly equivalent to a Bochner measurable function. Then, if $\mathbf{m}$ is the indefinite Pettis integral of $f$, defined by $\mathbf{m}(A)=\int_{A} f d \mu$, the associated cylindrical measure $\lambda=f(\mu)$ is not $\tau$-smooth [1, p. 672], so $\rho^{\prime}(f)(\omega)$ is not in $E$ for almost all $\omega$. In such a space $E$, every measure with bounded average range has a weak density, but such densities cannot always be obtained by liftings in the weak topology of $E$.

Examples of spaces satisfying the properties (a), (b), (c) include a nonseparable dual of a separable Banach space which does not have a subspace isomorphic to $l^{1}$, such as the dual of the James Tree space [6, Theorem 3].

\section{REFERENCES}

1. G. A. Edgar, Measurability in a Banach space, Indiana Univ. Math. J. 26 (1977), 663-677.

2. A. Goldman, Mesures cylindriques, mesures vectorielles et questions de concentration cylindrique, Pacific J. Math. 69 (1977), 385-413.

3. A. Ionescu Tulcea and C. Ionescu Tulcea, Topics in the theory of liftings, Ergebnisse der Mathematik und Ihrer Grenzgebiete, vol. 48, Springer-Verlag, Berlin and New York, 1969.

4. J. D. Knowles, Measures on topological spaces, Proc. London Math. Soc. 17 (1967), 139-156.

5. W. Moran, Measures and mappings on topological spaces, Proc. London Math. Soc. 19 (1969), 493-508.

6. K. Musial, The weak Radon-Nikodym property in Banach spaces, Studia Math. 64 (1979), 151-173.

7. J. C. Oxtoby, Measure and category, Graduate Texts in Mathematics, vol. 2, Springer-Verlag, Berlin and New York, 1971.

8. C. Sunyach, Une caractérisation des espaces universellement Radon-measurables, C. R. Acad. Sci. Paris Sér. A-B 268 (1969), 864-866.

9. V. S. Varadarajan, Measures on topological spaces, Amer. Math. Soc. Transl. (2) 48 (1965), 161-228.

Department of Mathematics, Ohio State University, Columbus, Ohio 43210

Équipe d'Analyse, Université Paris VI, 75230 Paris Cedex 05, France 